\title{
Diagnosis and Management of the Thyroid Nodules
}

\author{
Arturs Ozolins*,**, Zenons Narbuts*,**, Ilze Strumfa**, Peteris Prieditis***, Janis Gardovskis*,** \\ *Pauls Stradins Clinical University Hospital, Department of General Surgery \\ **Riga Stradins University, Latvia \\ *** Pauls Stradins Clinical University Hospital, Institute of Diagnostic Radiology
}

\begin{abstract}
Summary
Thyroid nodules are a common clinical problem. Most of the discovered nodules are benign, however increase in thyroid cancer incidence has been reported. For management of thyroid nodules distinction between benign and malignant thyroid tumors is essential. Thyroid nodule management involves notice to: clinical features, biochemical tests, imaging studies, fine needle aspiration, thyroid scintigraphy and immunocytochemistry. In spite of different combinations of diagnostic tools used, it is still difficult to precisely determine malignant potential of thyroid nodules.

Key words: thyroid nodules, FNA; immunocytochemistry, thyroid cancer.

Abbreviations: CT - Computed tomography; DTC - Differentiated thyroid cancer; FC - Follicular cancer; FNA - Fine needle aspiration; MEN 2 - Multiple endocrine neoplasia type 2; MNG - Multi nodular goiter; MRI - Magnetic resonance scanning; MTC - Medullary thyroid cancer; PTC - Papillary thyroid carcinoma; TPOAb - Anti-thyroid peroxidase antibody; TSH - Serum thyrotropin; US Ultrasound.
\end{abstract}

\section{INTRODUCTION}

Thyroid nodules are very common and are usually discovered during routine medical care. Nodular thyroid disease refers to the presence of a solid nodule, a multinodular gland, or one or more cystic lesions. It is estimated that $5-7 \%$ of adults have clinically detectable nodule in the thyroid and with the emergence of ultrasound (US) $20-67 \%$ of adults are diagnosed with one or more nodules in the thyroid (12). In autopsy series, the incidence of thyroid nodules in apparently normal thyroid glands is greater than 50\% (23). Thyroid nodules are more common in women and the prevalence is greater in countries affected by moderate or severe iodine deficiency, where goitre is endemic (3). External radiation also increases the incidence of thyroid nodules.

Most of the discovered nodules are benign, however, there are approximately 30,000 new cases of thyroid cancer annually in the US (17); the yearly incidence has increased from 3.6 per 100,000 in 1973 to 8.7 per 100,000 in 2002 and this tendency appears to be continuing (9). Accurate diagnosis of thyroid nodules is critical for proper management of nodules to distinguish the small proportion that are malignant from the very large proportion that are benign. Thyroid cancer represents $\sim 5-24 \%$ of thyroid nodules and $\sim 1-2 \%$ of all malignancies (15). Differentiated thyroid cancer (DTC), which includes papillary thyroid carcinoma (PTC) and follicular cancer (FC), constitutes the vast majority $(90 \%)$ of all thyroid cancers (27).

Many disorders, both benign and malignant, can cause thyroid nodules therefore exclusion of malignant thyroid lesions is clinically very important.

Usually, only nodules $>1 \mathrm{~cm}$ should be evaluated, because they have greater potential to be malignant.
However, there may be nodules $<1 \mathrm{~cm}$ that require evaluation, mostly due to suspicious US findings, associated lymphadenopathy, a history of neck irradiation, or a history of thyroid cancer in first-degree relatives.

Management involves attention to:

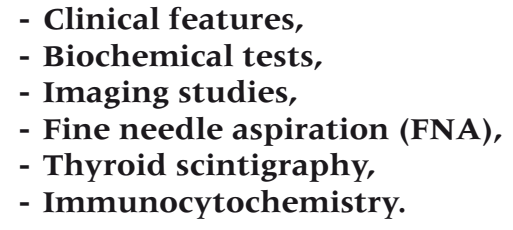

\section{Clinical Features.}

1.1. History. With the discovery of a thyroid nodule, a complete history and physical examination focused on the thyroid gland and cervical lymph nodes should be performed. Nonpalpable nodules have the same risk of malignancy as palpable nodules with the same size (14). The factors like young and old age, male gender, history of head and neck irradiation, family history of thyroid carcinoma, rapid growth and hoarseness can predict malignancy.

1.2. Symptoms and physical findings. Most patients with thyroid nodules have few or no symptoms. Usually no clear relationship exists between the histological features or size of the nodule and the reported symptoms. However, a large nodule can cause symptoms such as pressure, difficulty in swallowing and/or breathing, and a change in voice. Physical findings suggesting possible malignancy include vocal cord paralysis, lateral cervical lymphadenopathy, and tight relevance of the nodule to surrounding tissues. However, history and physical findings cannot differentiate most thyroid cancers from benign nodules. 


\section{Biochemical tests.}

2.1. Serum thyrotropin (TSH). With the discovery of a thyroid nodule, a TSH level should be obtained. The high sensitivity of the TSH assay for detecting even small thyroid dysfunction makes it the most useful test in the initial evaluation if thyroid nodules (29). Elevated serum TSH is associated with increased risk of malignancy in a thyroid nodule (4). If the serum TSH is subnormal, a thyroid scintigraphy using ${ }^{123}$ I should be obtained to document whether the nodule is hyperfunctioning, isofunctioning or nonfunctioning. Hyperfunctioning nodules rarely represent malignancy.

2.2. Measuring serum levels of free thyroid hormones and anti-thyroid peroxidase antibody (TPOAb) or anti-TSH-receptor antibody should be the next diagnostic step for confirmation of thyroid dysfunction if the TSH concentration is outside the reference range (1). In case of increased serum TSH free thyroxine and TPOAb should be tested to evaluate hypothyroidism. When the serum TSH is decreased free thyroxine and triiodothyronine should be assigned to evaluate hyperthyroidism. Most patients with a malignant thyroid nodule are clinically and biochemically euthyroid. This can be confirmed by normal levels of thyrotropin (TSH) and free thyroxine.

2.3. Routine assessment of serum thyroglobulin for initial evaluation of thyroid nodules is not recommended because it can be elevated in most thyroid diseases and is neither sensitive nor specific for thyroid cancer (8).

2.4. Calcitonin is a hormone that is produced in humans primarily by the parafollicular cells (C-cells) of the thyroid. The utilities of serum calcitonin assessment have been evaluated in a series of prospective, nonrandomized studies $(7,11)$. The use of routine serum calcitonin in patients with nodular thyroid disease may detect $\mathrm{C}$-cell hyperplasia and medullary thyroid cancer (MTC) at an earlier stage; however, routine testing in all patients with unselected thyroid nodules is still debated. Calcitonin levels can be increased in patients with pulmonary or pancreatic endocrine tumours, kidney failure or autoimmune thyroid disease; other factors are alcohol consumption and smoking. If calcitonin level is increased, the test should be repeated and, if confirmed in the absence of the above-mentioned modifiers, a pentagastrin stimulation test should be added to increase specificity (7). Measurement of serum calcitonin is mandatory in patients with family history or clinical suspicious of MTC or multiple endocrine neoplasia type 2 (MEN 2).

3. Imaging studies.

3.1. Ultrasound. High-resolution US is the most sensitive test available to detect thyroid lesions. It should be performed in all patients with suspected thyroid nodule or nodular goiter. Using US it is possible to diagnose pathological mass in the thyroid as well as the presence of cervical lymphadenopathy. The position, shape, size, margins, content, echogenic pattern and vascular features of the nodules can be described. Various sonographic characteristics associated with a higher likelihood of thyroid nodule malignancy have been evaluated in a series of studies $(6,21)$. The reported specificities for predicting malignancy are 41.4 - $92.2 \%$ for marked hypoechogenicity compared to normal thyroid parenchyma, 44.2 - 95\% for microcalcifications, $48.3-91.8 \%$ for irregular or microlobulated margins, and $\sim 80 \%$ for increased intranodular vascularity $(6,21)$. A shape taller than the width measured in the transverse dimension is an additional US pattern suggestive of malignant potential (6). Suspicious cervical lymphadenopathy is a specific but insensitive finding. No single sonographic feature or combinations of features are adequate to identify all malignant nodules; however, the coexistence of 2 or more suspicious US criteria greatly increases the risk of thyroid cancer (6). In patients with a normal thyroid on palpation and a low clinical risk of thyroid cancer US evaluation as a screening test is not recommended.

The most common sonographic appearances of PTC are a solid or predominantly solid and hypoechoic mass, usually with infiltrative irregular margins and increased nodular vascularity. Microcalcifications are highly specific for PTC. Whereas FC is more often iso - to hyperechoic with a thick irregular halo and lack of microcalcifications (16). FC that is $<2 \mathrm{~cm}$ in diameter usually has not been associated with metastatic disease. There are also certain sonographic criteria that may be predictive of a benign nodule. A pure cystic nodule or a nodule with spongiform appearance, defined as an aggregation of multiple microcystic components in more than $50 \%$ of the nodule volume, is specific for identification of benign thyroid nodule (21).

3.2. US elastography is a newly developed emerging and promising dynamic technique that has recently been applied in the diagnostic approach to nodular thyroid disease and has shown a high sensitivity and specificity in selected patients. It evaluates the degree of distortion of a tissue under application of an external force, based upon the principle that the softer parts of tissues deform more readily than the harder parts under compression, thereby allowing objective determination of tissue consistency (24). However, US elastography has some limitations because the nodule to be examined must be clearly distinguishable from other nodules. In addition, malignant lesions tend to be much harder than benign ones.

3.3. Magnetic resonance scanning (MRI) and computed tomography (CT) should not be used routinely in nodular thyroid disease because they are diagnostic for malignant lesions in very few cases (28). MRI and CT may be useful in assessment of size or substernal extension of a nodular goiter.

4. Fine needle aspiration (FNA) is the most accurate and cost - effective method for evaluating thyroid nodules, therefore clinical management of thyroid nodules should be guided by the combination of US evaluation and FNA biopsy. Cytological diagnosis is more reliable and the nondiagnostic rate is lower when FNA biopsy is performed with US guidance, because the most common cause of a false - negative cytological diagnosis is sampling error (30). Retrospective studies 
have reported lower rates of nondiagnostic and false negative cytology specimens from FNA performed by US guidance compared to palpation.

The FNA biopsy sample must be adequate for an interpretation that yields a low false - negative rate and should be reviewed by a cytopathologist with an interest in thyroid disease. The specimen is labelled "diagnostic" if it contains a minimum of 6 groupings of well preserved thyroid epithelial cells, consisting of at least 10 cells per group (25). The amount and type of colloid as well as the size of follicles should be described. For the diagnosis of PTC nuclear size, groove and intranuclear pseudoinclusions are important.

Five diagnostic categories are used: benign, nondiagnostic, follicular lesion, suspicious, and malignant. FNA results are classified as benign in 60-80\%; nondiagnostic in 10-15\%; follicular lesions in $10-20 \%$; suspicious in $2.5-10 \%$ and $3.5-10 \%$ are malignant $(25,30)$.

5. Thyroid scintigraphy is the only technique that allows for assessment of thyroid regional function. After radionuclide uptake, nodules may be classified as hyperfunctioning ("hot"), hypofunctioning ("cold"), or indeterminate. Nonfunctioning "cold" nodules are believed to have a probability of being malignant, but according to literature the reported malignancy risk is $3-15 \%$ (19). Hot nodules almost never represent clinically significant malignant lesions. Thyroid scintigraphy should be performed for a thyroid nodule or multi nodular goiter (MNG) if the TSH level is low or if a retrosternal goiter or ectopic thyroid tissue is suspected.

6. A growing number of immunocytochemical and molecular markers have been identified and tested with considerable variability in the outcomes of these studies. Today, in scientific publications many attempts have been described to find additional criteria to increase the accuracy of both surgical and FNA cytological specimens. These include galectin-3, galectin-7, HBME1, E-Cadherin, CD56, cytokeratin-19 and, Ki-67, P53 et cetera. Recent studies have confirmed the ability of HBME-1, E-Cadherin and CD56 to improve the accuracy of thyroid morphology $(10,22,26)$. It is likely that the combination of molecular markers will be used in the future to optimise management of patients with indeterminate cytology on FNA specimens.

\section{Management and therapy.}

7.1. A benign cytologic diagnosis is the most common finding in FNA which include colloid nodule, hyperplastic nodule, lymphocytic thyroiditis and benign cyst (13). If the nodule is benign on initial cytology, no immediate diagnostic procedures or treatment is further required. Most thyroid nodules with benign cytological results and no clinical and US risk factors require follow-up because of a low, but important falsenegative rate of up to $5 \%$ with FNA. It is recommended that all benign thyroid nodules be followed with clinical and US examination and TSH measurement 6-18 month after the initial FNA. Thyroid nodule growth is defined as more than $50 \%$ change in thyroid volume or a $20 \%$ increase in at least two nodule dimensions with a minimal increase of $2 \mathrm{~mm}$ in a solid nodule or in the solid portion of mixed cystic-solid nodules (5). If there is evidence of nodule growth or suspicious clinical or US changes, the repeated FNA should be performed and surgical resection should be considered.

Indications for surgical treatment in a patient with a benign thyroid nodule are as follows: neck pressure, dysphagia, and shortness of breath, a choking sensation, dyspnea, hoarseness or pain. Symptoms must be associated with the nodule or goiter and not with the pulmonary, cardiac or esophageal disorders or other disease.

In case of hyperfunctioning nodule or a multi nodular goiter surgical treatment or radioiodine is indicated. Before surgery euthyroid status has to be achieved. For benign uninodular goiter acceptable extend of surgery is lobectomy plus isthmectomy. In case of MNG (near) total thyroidectomy should be performed (20). Radioiodine therapy should be considered in case of hyperfunctioning nodule or a MNG. This method is best suited for patients with previous thyroid surgery or with the high surgical risk. Regular thyroid function measuring is mandatory because of possible presence of autoimmune thyroiditis or postradioiodine hypothyroidism. Absolute contraindications are pregnancy and breastfeeding.

7.2. FNA Specimens are nondiagnostic because of an insufficient number of follicular cells, which can be due to cyst fluid, bloody smears, or poor technique in preparing slides (13). For a nodule with an initial nondiagnostic cytology, reaspiration can be performed after a waiting period of at least 3 month (2). The reported malignancy rate in nondiagnostic specimens ranges $2-12 \%$. In case of repeated nondiagnostic FNA together with suspicious clinical and US findings, surgical resection should be performed. Some nodules, on the basis of auspicious clinical and US findings, may be followed up with close clinical and US control (18).

7.3. Follicular lesions category is used if definitive cytologic diagnosis of malignancy cannot be made and cytologic features indicate lesion of follicular nature. Follicular lesions appear as hypercellular specimens with microfollicular arrangement and decreased or absent colloid. Currently, no definitive morphologic criteria are available to distinguish benign from malignant follicular lesions. If nodule is classified as follicular neoplasm, repeated FNA is not recommended, while it is recommended in cases diagnosed as "atypical cells" to exclude a follicular neoplasm. About 20\% of such specimens are determined to be malignant in final histology. US features and elastography as well as molecular markers may provide adjunctive information and may improve the accuracy of cytologic diagnosis but they do not have consistent predictive value for malignancy. In most cases thyroid lobectomy and isthmectomy or total thyroidectomy, depending on clinical situation, with following histological examination is performed. Frozen section can be useful in case of non-total thyroidectomy. If clinical, cytological and US features are auspicious, clinical follow up can be 
considered without immediate diagnostic surgery.

7.4. Suspicious results in cytologic diagnosis are characterised by cytologic features suggesting but not fulfilling the criteria for a definitive diagnosis of malignancy. In suspicious results there are also included samples with poor cellularity and preservation but clear signs indicating malignancy. Frozen section is usually recommended for surgical decision.

7.5. Malignant results include samples characterised by malignant cytologic features that are reliably identified by cytopathologist. Preferably type of carcinoma should be stated in cytologic report. The most frequent malignant lesion is PTC and if a cytology result is diagnostic of or suspicious for PTC, thyroidectomy by the surgeon experienced in endocrine surgery is recommended. Malignant lesions also include MTC, anaplastic carcinoma, lymphoma, metastatic cancers and miscellaneous thyroid tumours. For anaplastic carcinoma, lymphoma and metastatic cancers, further diagnostic workup is recommended before surgery. In selected cases CT and MRI may be performed.

\section{CONCLUSIONS}

Thyroid nodules are usually benign. However, proper diagnostic workup is important to identify the few malignant cases. Thyroid US and FNA are well established techniques recommended making the correct clinical and pathological diagnosis. There are specific US characteristics associated with a higher likelihood of thyroid nodule malignancy. The combination of molecular markers appears to be useful to optimise management of patients with indeterminate cytology on FNA specimens. It is still difficult to precisely determine malignant potential of thyroid nodules despite the allavailable diagnostic tools.

\section{Conflict of interest: None}

\section{REFERENCES}

1. Baloch Z, Carayon $\mathrm{P}$, et al. Laboratory medicine practice guidelines. Laboratory support for the diagnosis and monitoring of thyroid disease // Thyroid, 2003; 13(1):3 - 126

2. Baloch ZW, LiVolsi VA, et al. Diagnostic terminology and morphologic criteria for cytologic diagnosis of thyroid lesions: a synopsis of the National Cancer Institute Thyroid Fine-Needle Aspiration State of the Science Conference // Diagn Cytopathol, 2008; 36(6): $425-437$

3. Belfiore A, La Rosa GL, et al. Cancer risk in patients with cold thyroid nodules: relevance of iodine intake, sex, age, and multinodularity // Am J Med, 1992; 93(4):363 - 369

4. Boelaert K, Horacek J, et al. Serum thyrotropin concentration as a novel predictor of malignancy in thyroid nodules investigated by fine-needle aspiration // J Clin Endocrinol Metab, 2006; 91(11):4295 - 4301

5. Brauer VF, Eder $\mathrm{P}$, et al. Interobserver variation for ultrasound determination of thyroid nodule volumes // Thyroid, 2005; 15(10):1169-1175
6. Cappelli C, Castellano $\mathrm{M}$, et al. The predictive value of ultrasound findings in the management of thyroid nodules // QJM, 2007; 100(1):29 - 35

7. Costante $G$, Meringolo D, et al. Predictive value of serum calcitonin levels for preoperative diagnosis of medullary thyroid carcinoma in a cohort of 5817 consecutive patients with thyroid nodules // J Clin Endocrinol Metab, 2007; 92(2):450 - 455

8. Date J, Feldt-Rasmussen U, et al. Long-term observation of serum thyroglobulin after resection of nontoxic goiter and relation to ultrasonographically demonstrated relapse // World J Surg, 1996; 20(3):351 - 356

9. Davies L, Welch HG. Increasing incidence of thyroid cancer in the United States, 1973-2002 // JAMA, 2006; 295(18):2164-2167

10. El Demellawy D, Nasr A, et al. Application of CD56, P63 and CK19 immunohistochemistry in the diagnosis of papillary carcinoma of the thyroid // Diagn Pathol, 2008; 3:5

11. Elisei R, Bottici V, et al. Impact of routine measurement of serum calcitonin on the diagnosis and outcome of medullary thyroid cancer: experience in 10,864 patients with nodular thyroid disorders // J Clin Endocrinol Metab, 2004; $89(1): 163-168$

12. Gharib H, Goellner JR. Fine-needle aspiration biopsy of thyroid nodules // Endocr Pract, 1995; $1(6): 410-417$

13. Goellner JR, Gharib H, et al. Fine needle aspiration cytology of the thyroid, 1980 to 1986 // Acta Cytol, $1987 ; 31(5): 587-590$

14. Hagag P, Strauss S, et al. Role of ultrasound-guided fine-needle aspiration biopsy in evaluation of nonpalpable thyroid nodules // Thyroid, 1998; 8(11):989- 995

15. Hundahl SA, Fleming ID, et al. A National Cancer Data Base report on 53,856 cases of thyroid carcinoma treated in the U.S., 1985-1995 // Cancer, 1998; 83(12):2638 - 2648

16. Jeh SK, Jung SL, et al. Evaluating the degree of conformity of papillary carcinoma and follicular carcinoma to the reported ultrasonographic findings of malignant thyroid tumor // Korean J Radiol, 2007; 8(3):192 - 197

17. Jemal A, Siegel R, et al. Cancer statistics, 2006 // CA Cancer J Clin, 2006; 56(2):106 - 130

18. Kwak JY, Kim EK, et al. How to combine ultrasound and cytological information in decision making about thyroid nodules // Eur Radiol, 2009; 19(8):1923 - 1931

19. La Rosa GL, Belfiore A, et al. Evaluation of the fine needle aspiration biopsy in the preoperative selection of cold thyroid nodules // Cancer, 1991; 67(8):2137 - 2141

20. Moalem J, Suh I, et al. Treatment and prevention of recurrence of multinodular goiter: an evidencebased review of the literature // World J Surg, 2008; 32(7):1301-1312 
21. Moon WJ, Jung SL, et al. Benign and malignant thyroid nodules: US differentiation-multicenter retrospective study // Radiology, 2008; 247(3): $762-770$

22. Ozolins A, Narbuts Z, et al. Diagnostic utility of immunohistochemical panel in various thyroid pathologies // Langenbecks Arch Surg, 2010; [Epub ahead of print]

23. Pacini F, Burroni L, et al. Management of thyroid nodules: a clinicopathological, evidence-based approach // Eur J Nucl Med Mol Imaging, 2004; 31(10):1443 - 1449

24. Rago T, Santini F, et al. Elastography: new developments in ultrasound for predicting malignancy in thyroid nodules // J Clin Endocrinol Metab, 2007; 92(8):2917 - 2922

25. Redman $\mathrm{R}$, Zalaznick $\mathrm{H}$, et al. The impact of assessing specimen adequacy and number of needle passes for fine-needle aspiration biopsy of thyroid nodules // Thyroid, 2006; 16(1):55 - 60

26. Saleh HA, Feng J, et al. Differential expression of galectin-3, CK19, HBME1, and Ret oncoprotein in the diagnosis of thyroid neoplasms by fine needle aspiration biopsy // Cytojournal, 2009; 6:18
27. Sherman SI. Thyroid carcinoma // Lancet, 2003; 361 (9356):501 - 511

28. Shetty SK, Maher MM, et al. Significance of incidental thyroid lesions detected on CT: correlation among CT, sonography, and pathology // AJR Am J Roentgenol, 2006; 187(5):1349 - 1356

29. Spencer CA, Takeuchi M, et al. Current status and performance goals for serum thyrotropin (TSH) assays // Clin Chem, 1996; 42(1):140 - 145

30. Wu HH, Jones JN, et al. Fine-needle aspiration cytology of the thyroid: ten years experience in a community teaching hospital // Diagn Cytopathol, $2006 ; 34(2): 93-96$

\section{Address:}

Arturs Ozolins,

Pauls Stradins Clinical University Hospital

Department of Surgery

Pilsonu street 13, Riga, LV-1002

E-mail: artursozolins1@gmail.com 